\title{
Flexibility and local structure of a worm-like cylinder of self-assembled discotic triazine triamide
}

\author{
Yasuya Kudo ${ }^{1}$, Mina Sakuragi ${ }^{1}$, Satoshi Hashida ${ }^{1}$, Rempei Kuwahara ${ }^{2}$, Tsutomu Ishi- $\mathrm{I}^{2}$, Hiroyasu Masunaga ${ }^{3}$ \\ and Kazuo Sakurai ${ }^{1}$ \\ Small- and wide-angle X-ray scattering from an organogel system made from discotic triazine triamide (TRI) in octanol was \\ carried out using a synchrotron X-ray source to provide scattering profiles for a wide range of scattering vectors, of range \\ $0.050 \mathrm{~nm}^{-1}<\boldsymbol{q}<20 \mathrm{~nm}^{-1}$, where $q$ is the magnitude of the scattering vector. The analyses of the profiles revealed the \\ following features: (1) the amorphous nature of the molecular stacking at a distance of $0.32 \mathrm{~nm}$; (2) the presence of a \\ semiflexible rod-like scattering object with a radius of $1.3 \mathrm{~nm}$ and a Kuhn length of $55 \mathrm{~nm}$; and (3) branching of the cylinder \\ formed in the gel state. These findings lead to the conclusion that the aggregated TRI functions in the manner of a semiflexible \\ polymer chain in octanol and that the polymer-chain-like behavior of TRI cylinders is the origin of the gel nature of this system. \\ Polymer Journal (2010) 42, 812-817; doi:10.1038/pj.2010.72; published online 25 August 2010
}

Keywords: discotic triazine triamide; organogel; small- and wide-angle X-ray scattering

\section{INTRODUCTION}

Organogelators are a class of compounds that can gelatinize particular organic solvents or increase their viscosity when they are added even in small concentrations of $<1-2 \mathrm{wt} \% .^{1,2}$ In the gel state, organogelator molecules self-assemble to form fine fibrils, and these elemental fibrils tend to bundle with each other to form micrometer-scale cylinders or network structures. ${ }^{3-5}$ The mixture's entire morphology becomes sponge like and is thus able to absorb a large amount of solvent. This is the reason that this system functions as a gel or viscoelastic material; such compounds are called organogelators. As they constitute an important class of materials for their applications in template materials and drug delivery systems, an enormous number of studies have been devoted to the synthesis of new gelators and to the exploration of their fundamental properties. Although organogelators have been studied extensively, there are only a few reports on the fundamental morphological and structural properties of the elemental fibrils. ${ }^{4}$

Synchrotron X-ray scattering is one of the most powerful tools for investigating the fibrillar structure of organogelators. ${ }^{4,6-8}$ As a synchrotron X-ray source is $>10^{6}$ times more intense than conventional $\mathrm{X}$-ray sources, it is immensely advantageous for investigating dilute solution systems, such as organogels. Terech et al. , $^{4,5,9}$ measured small-angle X-ray scattering (SAXS) from several organogels and found that most of the scattering data can be fitted by a randomly oriented long cylinder model. Some studies showed that the cylinder radii are much larger than the constituting gelator molecules, suggest- ing the presence of a hierarchical structure. To clarify such hierarchical structures of organogels and to analyze how gelator molecules selfassemble inside the cylinder, measurements of scattering intensity over a wide range of scattering angles is required. It should be noted that, for some cases, crystalline peaks are observed in the wide-angle scattering (WAXS) region. ${ }^{6}$ These types of assemblies should be classified as needle-like crystals rather than as organogels. For this reason, WAXS combined with SAXS is important to understand the nature of the self-assembly of organogels.

Certain types of gelators can form columnar or ribbon-like supramolecular structures by using intermolecular interactions (such as hydrogen bonding) between functional groups, such as amide, carboxyl and hydroxyl groups. In this paper, we report our studies on a series of discotic triazine triamide (TRI) organogelators (see the chemical structure in Figure 1a). ${ }^{10}$ One of the interesting features of TRI organogelators is that when a small amount of chiral TRI is added to a large amount of achiral TRI, all supramolecular aggregates show only one handedness of the helix. This chiral amplification can be explained with the model that TRI molecules stack with each other to form a helical columnar supramolecule, so that three-sided chains are arranged alternately to avoid steric hindrance, as presented in Figure 1b. However, whether TRI molecules do form such a cylinder has not been confirmed, and proving this hypothesis is important for understanding the molecular nature and design of other functional materials. The main aim of this study was to explore the supramolecular structure of the TRI system by combining WAXS

${ }^{1}$ Department of Chemistry and Biochemistry, Faculty of Engineering, The University of Kitakyushu, Fukuoka, Japan; ${ }^{2}$ Institute for Materials Chemistry and Engineering (IMCE), and Interdisciplinary Graduate School of Engineering Sciences, Kyushu University, Fukuoka, Japan and ${ }^{3}$ SPring-8, Japan Synchrotron Radiation Research Institute (JASRI), Hyogo, Japan

Correspondence: Professor K Sakurai, Department of Chemistry and Biochemistry, Faculty of Engineering, The Unviersity of Kitakyushu, 1-1, Hibikino, Wakamatu-ku, Kitakyushu, Fukuoka 808-0135, Japan.

E-mail: sakurai@env.kitakyu-u.ac.jp

Received 1 March 2010; revised 12 May 2010; accepted 23 June 2010; published online 25 August 2010 
a

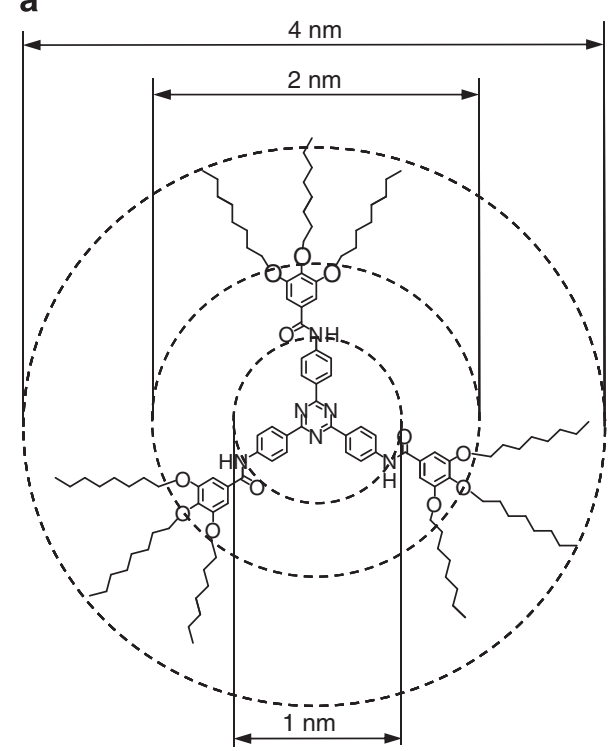

b

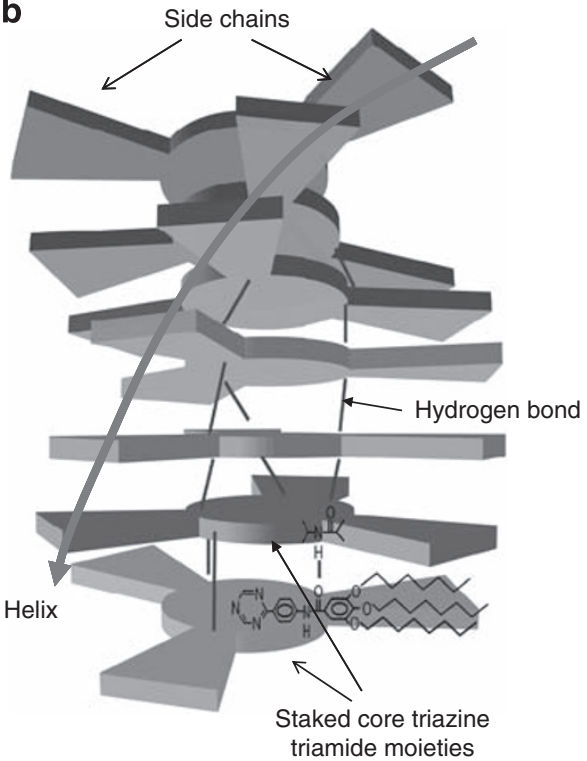

Figure 1 (a) Chemical structure of 2,4,6-tris(4-(3,4,5-tris(octyloxyphenyl)amino)phenyl)-1,3,5-triazine, denoted by TRI-C8. (b) A proposed model for the molecular assembly of TRI-C to explain the sudden emergence of chiral amplification when a small amount of TRI-C8 analog that has a chiral center in the side chain is added. In the stacked cylinder, three bulky side chains of TRI-C are nested with each other to avoid steric hindrance, causing helical stacking. The driving force of the stacking is the hydrogen bonds between the adjacent amide groups and the $\pi-\pi$ interaction between the core triazine moieties.

and SAXS measurements over a wide range of $q$ and clarify how the molecule self-assembles in the gel.

\section{EXPERIMENTAL PROCEDURE}

\section{Sample preparation}

We synthesized one of the discotic compounds, 2,4,6-tris(4-(3,4,5-tris (octyloxyphenyl)amino)phenyl)-1,3,5-triazine (see the chemical structure in Figure la and denoted by TRI-C8), according to a method described elsewhere, ${ }^{10}$ and prepared $1.0 \mathrm{~mm}\left(1.8 \times 10^{-4} \mathrm{wt} \%\right), 2.5,5.0,10.0,25.0 \mathrm{~mm}$ and $50 \mathrm{~mm}\left(9.1 \times 10^{-3} \mathrm{wt} \%\right)$ octanol solutions. The $1-5 \mathrm{~mm}$ samples were solutions with low viscosity at room temperature, whereas the 10, 25 and $50 \mathrm{~mm}$ samples were highly viscoelastic. When a vial containing the $50 \mathrm{~mm}$ solution was left upside down for a few hours at room temperature, the solution eventually flowed, suggesting that the TRI-C8 system is not in gel state for long time scales. The samples were loaded into a quartz cell (MarkRohrchen, West Berlin, Federal Republic of Germany) with a $2 \mathrm{~mm}$ diameter, and the cell was sealed with an epoxy adhesive. ${ }^{8}$

\section{X-ray scattering}

X-ray scattering experiments were carried out at the BL40B2 station at the SPring-8 facility in Japan. The gelator concentration $(C)$ dependence of the SAXS intensity $(I)$ was measured with a Rigaku R-AXIS IV++ system (RIGAKU Co., Tokyo, Japan, a $30 \times 30 \mathrm{~cm}$ imaging plate). The exposure time ranged from 5 to $20 \mathrm{~min}$, depending on the concentration. Three distances from the sample to the detector $\left(\mathrm{SDD}=4.1 \times 10^{2} \mathrm{~cm}\right.$ for USAXS, $3.1 \times 10^{2} \mathrm{~cm}$ for SAXS and $40 \mathrm{~cm}$ for WAXS, where $U$ stands for 'ultra') were chosen to cover the range $0.030 \mathrm{~nm}^{-1}<q<20 \mathrm{~nm}^{-1}$, where $q$ is the magnitude of the scattering vector, which corresponds to real distances in the range $0.31-2.1 \times 10^{2} \mathrm{~nm}$. The detailed experimental setup in the present beamline and the method for combining SAXS and WAXS data have been described elsewhere. ${ }^{11}$

Every sample was heated to about $80^{\circ} \mathrm{C}$, then slowly cooled to room temperature about $24 \mathrm{~h}$ before the measurement to erase thermal hysteresis. The two-dimensional scattering pattern consisted of concentric circles, and the $I$ vs $q$ profile was independent of the azimuthal angle. Therefore, we took a circular average of $I$, and the resultant $I$ vs $q$ plot was used for discussion. We confirmed that the degradation of samples was negligibly small during exposure to the synchrotron X-ray by comparing SAXS profiles before and after exposure for $200 \mathrm{~s}$. As there was no concentration dependence of the WAXS scattering, we measured WAXS only from the $50 \mathrm{~mm}$ octanol solutions.

The excess scattering from the solutions was obtained by subtracting $I(q)$ of octanol from that of each solution using the following equation:

$$
I(q)=\frac{I_{\text {sample }}(q)}{T_{\text {sample }} \times X_{\text {in }}}-\frac{I_{\text {solvent }}(q)}{T_{\text {solvent }} \times X_{\text {in }}}
$$

Here, $T$ and $X_{\text {in }}$ are the transmittance and the read value of the ion chamber in front of the sample, respectively. The resultant $I(q)$ data obtained from different SDD setups were superimposed with each other to obtain an $I(q)$ profile for the entire $q$ range. As presented in Supplementary information, because of the large background from the solvent and the window material used in the setup, for the two $q$ ranges $3.5-4.5 \mathrm{~nm}^{-1}$ and $12-17 \mathrm{~nm}^{-1}$, the complete subtraction using equation (1) was difficult.

\section{Transmission electron microscopy}

Transmission electron microscopy (TEM) was carried out with a JEM-3010 (JEOL Ltd., Tokyo, Japan) in bright field mode with a $200 \mathrm{kV}$ accelerating voltage. The $50 \mathrm{~mm}$ octanol gel solution was placed on a TEM grid (mesh size: $1.5-8 \mu \mathrm{m}$, STEM $150 \mathrm{Cu}$, where S stands for 'scanning'), and the grid was freeze-dried. Before the observation, the sample was stained with a phosphotungstic acid water solution $(0.5 \mathrm{wt} \%)$.

\section{RESULTS AND DISCUSSION}

\section{Entire scattering profile of TRI-C8}

Figure 2a plots $I(q) / C$ against $q$ in the range of $0.032 \mathrm{~nm}^{-1}<q$ $<7.5 \mathrm{~nm}^{-1}$ at room temperature for the solution of $C=1.0 \mathrm{~mm}$ after overlaying SAXS and USAXS data, where $C$ is the TRI-C8 molar concentration. No appreciable concentration (or dense packing ${ }^{12}$ ) effect was observed in the scattering intensity at this concentration. For $q<1 \mathrm{~nm}^{-1}, I(q) / C$ follows the relation $I(q) \propto q^{-1}$, indicating the presence of a rod-like scattering object. To fit the data, we applied the following form factor for a solid cylinder with the radius of $R$ :

$$
I(q) \propto \frac{1}{q}\left[J_{1}(q R) / q R\right]^{2}
$$


a

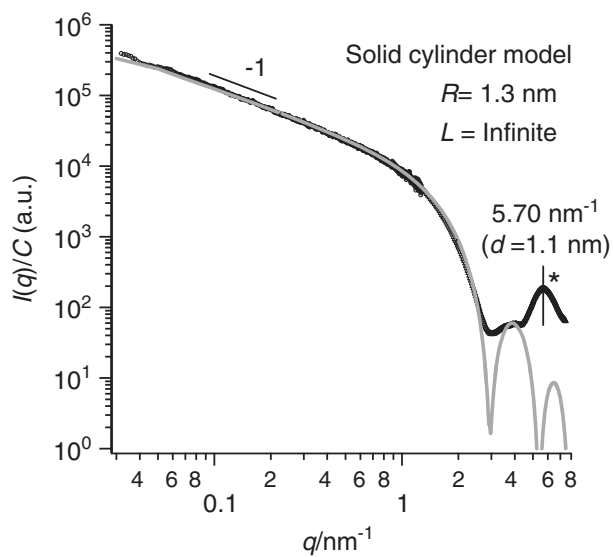

b

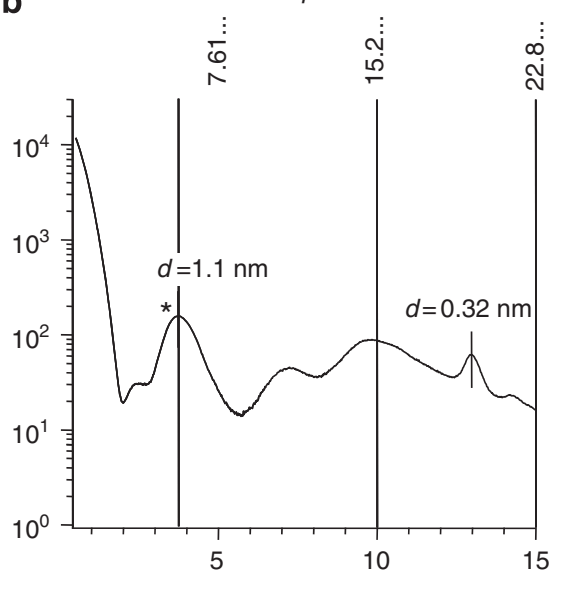

Figure 2 The entire scattering profile of TRI-C8 in octanol. (a) Superimposed SAXS and USAXS data for TRI-C8 for $C=1.0$ mM at room temperature; (b) WAXS region for $C=50 \mathrm{~mm}$. Asterisks indicate identical peaks.

Here, $J_{1}$ is a Bessel function of the first kind. The best-fit curve is presented in the figure with a gray solid line, which shows that the data can be reasonably represented assuming a solid cylinder scattering body with $R=1.3 \pm 0.05 \mathrm{~nm}$, in the range of $0.3 \mathrm{~nm}^{-1}<q$ $<5 \mathrm{~nm}^{-1}$. Referring to the chemical structure and its size presented in Figure 1a, the value of $R=1.3 \mathrm{~nm}$ is related to the aromatic moieties of TRI-C8. The precision error in determining the diameter of $1.3 \mathrm{~nm}$ was estimated to be within $0.05 \mathrm{~nm}$ of the cross-sectional Guinier plot (that is, $\ln [I(q) q]$ vs $q^{2}$ ) in the $0.03 \mathrm{~nm}^{-1}<q<2 \mathrm{~nm}^{-1}$ region. To our knowledge, there have been very few cases of organogels that can be fitted by a simple cylinder model in such a wide $q$ range.

We could fit the data with a core-shell cylinder model, for example, with a core radius of $0.5 \mathrm{~nm}$ and a shell radius of $1.3 \mathrm{~nm}$ with an appropriate electron density difference between the core and shell. Such models could fit the experimental data with almost the same accuracy as the solid cylinder model. However, the excellent reproduction in Figure 2a indicates that there is no need to apply a more elaborate mode. Owing to the chemical similarity between the TRI-C8 alkyl side chain and the solvent octanol, there should be hardly any difference in the electron density between them, and thus we could fit the data with a solid cylinder of almost the same size as that of the central aromatic moieties of TRI-C8. When we carefully examined the low $q$ region, the data points deviated upward from the mode, which can be ascribed to the flexibility of the cylinder and will be discussed later.

At $5 \mathrm{~nm}^{-1}<q$ in panel a, the data points deviate from the cylinder model. There is a clear peak at $5.70 \mathrm{~nm}^{-1}(D=1.1 \mathrm{~nm}$, where $D$ is the Bragg distance). Figure $2 \mathrm{~b}$ presents the WAXS results for $C=50 \mathrm{~mm}$ (there is no concentration dependence in this range). We did not observe any sharp crystalline peaks in this region, indicating the amorphous nature of the assembling. Although there are large experimental errors due to the presence of the intense peak from the solvent at $7 \mathrm{~nm}^{-1}<q<20 \mathrm{~nm}^{-1}\left(5^{\circ}<2 \theta<15^{\circ}\right)$, when we carefully subtracted the solvent scattering from the sample, we observed a rather clear peak at $q=19.7 \mathrm{~nm}^{-1}(D=0.32 \mathrm{~nm})$ and two broad peaks at $q=11.3$ and $14.5 \mathrm{~nm}^{-1}$, as well as the $5.70 \mathrm{~nm}^{-1}$ peak. One reason for the use of the rather concentrated $50 \mathrm{~mm}$ solution is to facilitate the subtraction of the solvent peak. As presented in the supporting information, because the $14.5 \mathrm{~nm}^{-1}$ peak was overlaid by the solvent peak, the actual intensity of this peak is ambiguous.
The value of $D=0.32 \mathrm{~nm}$ is expected to be the $\pi-\pi$ stacking distance between the discotic triazine moieties, which explains the peak at $q=19.7 \mathrm{~nm}^{-1}$. The $11.3 \mathrm{~nm}^{-1}$ peak may be assigned to the second diffraction peak of the $5.70 \mathrm{~nm}^{-1}$ peak; however, the structural origin of these peaks is not clear. One possible explanation is as follows. The $D$ value of the $5.70 \mathrm{~nm}^{-1}$ peak is almost three times that of the $19.6 \mathrm{~nm}^{-1}$ peak, suggesting that the origin of the $5.70 \mathrm{~nm}^{-1}$ peak can be ascribed to the helix pitch of the stacking. If this explanation is correct, we can speculate that TRI-C8 forms a $33_{1}$ helix (that is, three molecules are present in one helix), which is consistent with the molecular model presented by Ishi-i et al. ${ }^{10}$ The other peak around $q=14.5 \mathrm{~nm}^{-1}$ can be ascribed to the overlapping of the third diffraction peak at $q=5.7 \mathrm{~nm}^{-1}$ and some other local structures from the side chains.

\section{Concentration dependence of the intensity at the low $q$ region}

Figure 3 compares the concentration dependence of $I(q) / C$ for five different samples in the USAX range, where the $I(q) / C$ values at the limit of $C \rightarrow 0$ were obtained by graphical extrapolation, as presented in the supporting information. There seems to be three regions in the low $q$ scattering: good agreement with a solid cylinder model ( $\mathrm{C}$ region), downward deviation from the slope of -1 (B region) and upward curvature in the low $q$ (A region). The downward deviation becomes prominent as the concentration increases, indicating that this is caused by the inter-cylinder diffraction, the so-called dense packing effect. The upward region is clearly noticeable for $C=1.0,10$ and $25 \mathrm{~mm}$, and seems to disappear at $C=2.5$ and $5 \mathrm{~mm}$, which indicates that the origin of this phenomenon should be different between the concentrated $(C>10 \mathrm{~mm})$ and diluted $(C$ $<2.5 \mathrm{~mm}$ ) solutions. According to the scattering theory, such an upward scattering can be ascribed to the flexibility of the cylindrical objects, to branching or large aggregates. As the 10 and $25 \mathrm{~mm}$ solutions showed gelation, the upward curvature for the concentrated solutions can be ascribed to the branching of the cylinders, as proposed by Terech et al. ${ }^{4}$

The upward curvature in the diluted solution (these were not in the gel state) can be ascribed to the flexibility. For $C=1.0,2.5$ and $5 \mathrm{~mm}$, we extrapolated $I(q) / C$ to $C=0$ (see the Supporting information S2) and constructed a plot of $q I(q) / C$ against $q$ (a Holtzer plot) in Figure 4 


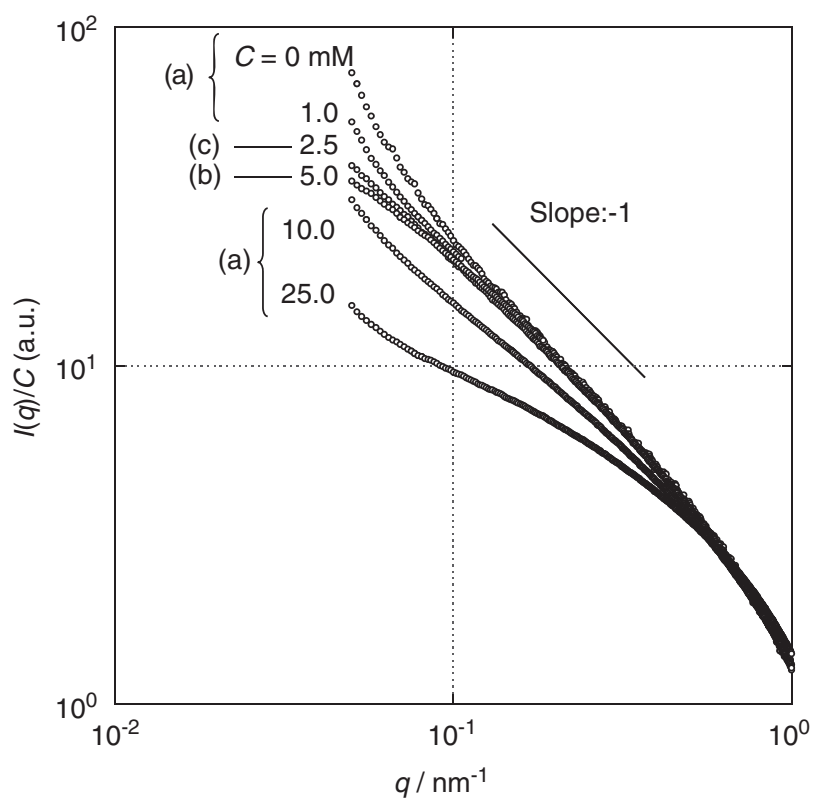

Figure 3 Concentration dependence of the scattering profiles in the low-angle region. (a) Upward curvature region $(C<2.5$ and $C>5)$ related either to the branching of the cylinder in the concentrated solution in the gel state or to the flexibility of the cylinder. (b) Downward deviation (around $5.0 \mathrm{~mm}$ ) from the rod limit due to the dense packing effect. (c) Rod scattering (around $2.5 \mathrm{~mm}$ ).

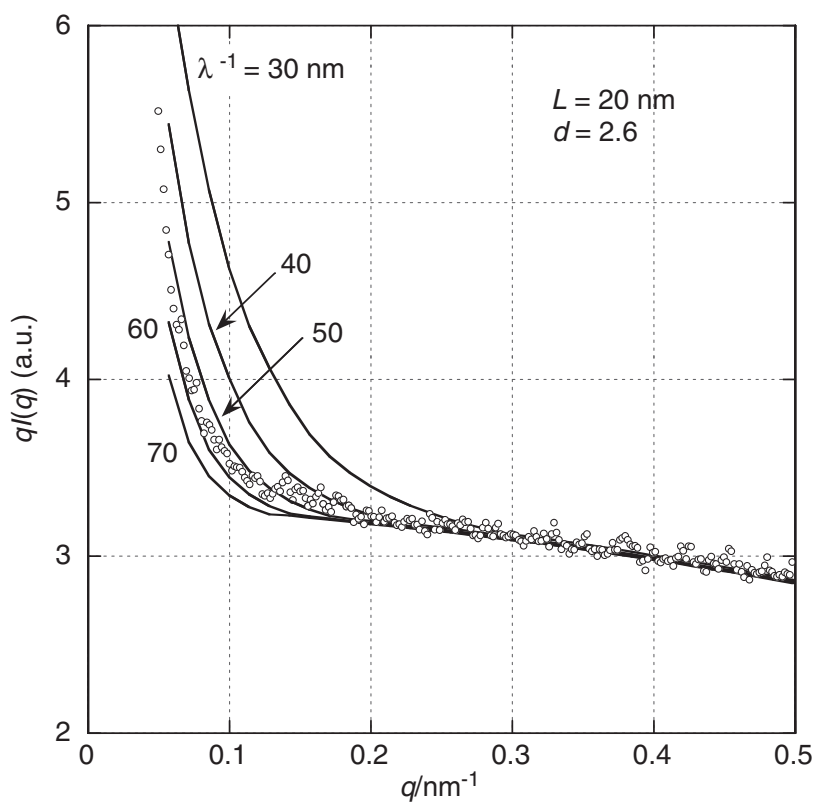

Figure 4 The Holzer plot comparing the extrapolated $/(q)$ with $C=0$ and the calculated values from the semiflexible worm-like cylinder with $R=1.3 \mathrm{~nm}$, $L=200 \mathrm{~nm}$ and $\lambda^{-1}=30-70 \mathrm{~nm}$.

(unfilled circles). This extrapolation should remove the dense packing effect from the data and elucidate the form factor from the data. Nakamura and Norisuye calculated the form factor for the KratkyPorod worm-like chain, which is the most appropriate model for semiflexible cylinders.
According to their theory ${ }^{13}$, when the flexibility of cylinders has to be taken into account, that is, the $q$ dependence of $I(q)$ transitions from $I(q) \propto q^{-1}$ to $\propto q^{-2}$ with decreasing $q$, rigid cylinder models cannot be used to fit the data points. The form factor for a cylindrical Kratky-Porod chain (called a worm-like cylinder) may be expressed by the following equation:

$$
I(q) / C_{\mathrm{D}}=L^{-2} \int_{0}^{L}(L-x) F(\vec{q} ; x) \mathrm{d} x
$$

Here, $F(\vec{q} ; x)$ can be expressed as the Fourier transform of the probability density function that the contour point $x$ is found at a specified point $\vec{r}$ (which can be related to $\vec{q}$ ) and may be expanded with the moments $\left\langle\vec{r}^{2 n}\right\rangle$. Nakamura and Norisuye calculated these moments for the Kratky-Porod chain and obtained a series of numerical tables that allow us to calculate the form factor for the entire $q$ range as a function of $q$, the Kuhn segment length $\left(\lambda^{-1}\right)$, the length of the cylinder $L$ and the radius of the single layer cylinder $R$. To our knowledge, their theory can reproduce the $q$ dependence of $I(q)$ in the transition region better than other theories.

The solid lines in Figure 4 are calculated values from the Nakamura-Norisuye theory with a fixed $L=200 \mathrm{~nm}$ and varying $\lambda^{-1}$ from 30 to $70 \mathrm{~nm}$. When we fitted the data with the theory, we shifted the calculated lines to overlap in the range of $q>0.3-2 \mathrm{~nm}^{-1}$. We fixed the cylinder length at $L=200 \mathrm{~nm}$ for convenience; this length can be considered long enough to ignore the chain length effect (that is, the cylinder can be considered to have infinite length).

Comparing the data with the calculations, we can evaluate $\lambda^{-1}$ of TRI-C8 in octanol to be $55 \pm 5 \mathrm{~nm}$. When we compare this value with that of other stiff polymer chains, such as $20-40 \mathrm{~nm}$ for poly $(n$-hexyl isocyanate), $60 \mathrm{~nm}$ for DNA double helix and $400 \mathrm{~nm}$ for schizophyllan (triple helix of $\beta$-1,3-glucan), the present system is not as rigid as expected. This may be because of the fact that each TRI-C8 molecule is connected with the hydrogen bonds and hydrophobic interactions that are weaker than covalent bonds. When we increased the temperature to $100{ }^{\circ} \mathrm{C}$, we did not observe any influence from the finite cylinder length in the examined $q$ range.

The temperature dependence of the scattering profiles of the 5 and $25 \mathrm{~mm}$ solutions is presented in Figures $5 \mathrm{a}$ and $\mathrm{b}$, respectively. With increasing temperature, the upward curvature in the low $q$ region becomes less notable (panel b) and the intensity drop around $q=3 \mathrm{~nm}^{-1}$ becomes small (panel a). The latter phenomenon can be interpreted by considering the fact that the distribution of the cylinder radius increases with increasing temperature, probably because of the increasing thermal motion of the stacked TRI-C8 molecules. When the temperature was increased, the $25 \mathrm{~mm}$ solution showed the gel-sol transition. Accordingly, the upward curvature in the low $q$ disappeared, indicating a decrease of the network to maintain the gel.

\section{TEM and dynamic viscosity measurements}

Figure 6 presents the frequency dependence of the storage and loss moduli $\left(G^{\prime}\right.$ and $\left.G^{\prime \prime}\right)$ for the $25 \mathrm{~mm}$ solution. The magnitude of $G^{\prime}$ is always larger than that of $G^{\prime \prime}$ in the range of $0.1-100 \mathrm{rad} \mathrm{s}^{-1}$, indicating the elastic nature of the TRI-C8/octanol solution. This feature is consistent with the presence of the branching of the cylinder. Figure 7 presents a typical image for a freeze-dried gel of TRI-C8. The image consists of entangled long flexible cylinders with diameters of about $4 \mathrm{~nm}$. This value is consistent with the SAXS results and also with that from the one molecular size. The presence of branched cylinders is consistent with the upward scattering in the concentrated solutions. 

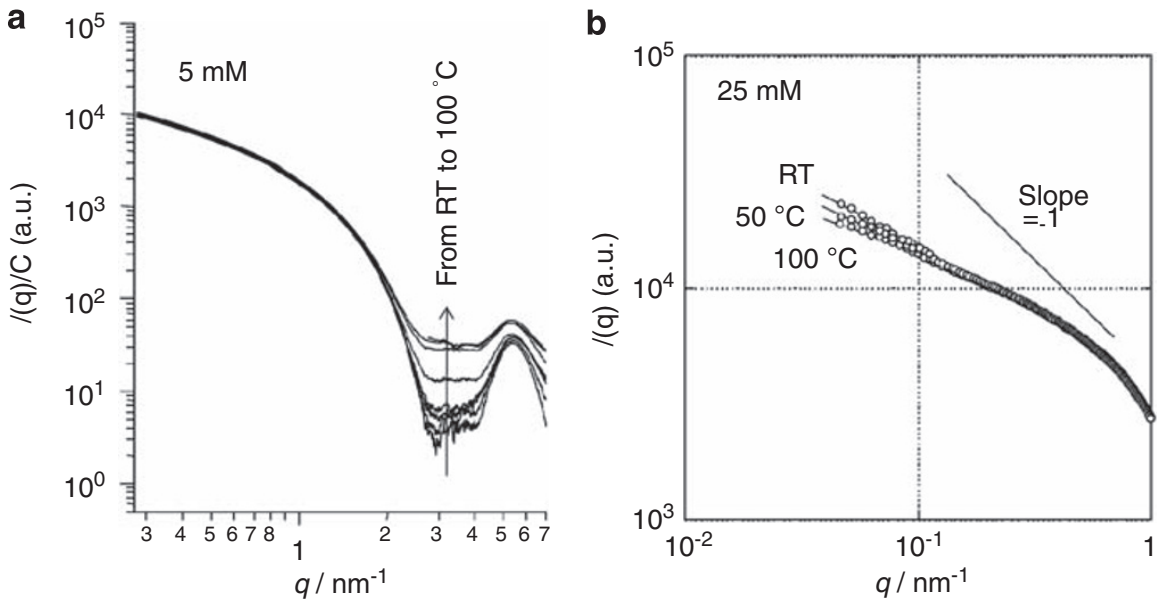

Figure 5 Temperature dependence of the scattering profiles for SAXS at $C=5 \mathrm{~mm}$ (a) and for USAX for $C=25 \mathrm{~mm}$ (b).

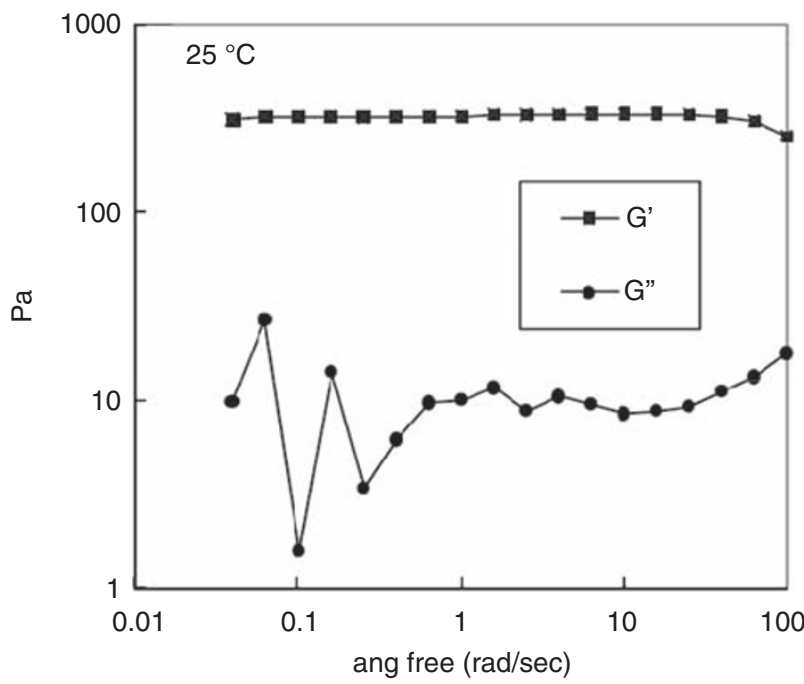

Figure 6 Dynamic viscosity measurements for $C=25 \mathrm{~mm}$ at room temperature.

\section{CONCLUSIONS}

Synchrotron X-ray scattering was carried out in TRI-C8/octanol solutions in the range of $0.050 \mathrm{~nm}^{-1}<q<20 \mathrm{~nm}^{-1}$, corresponding to distances in real space in the range of $0.31-1.26 \times 10^{2} \mathrm{~nm}$. The WAXS measurements suggested the presence of helical structures and the amorphous nature of the stacked molecules, with a spacing of $0.32 \mathrm{~nm}$. The SAXS and USAXS data can be fitted with a semiflexible cylinder model with $R=1.3 \mathrm{~nm}$ and $\lambda^{-1}=55 \pm 5 \mathrm{~nm}$. As the concentration increased, the USAXS intensities deviated downward from the semiflexible cylinder model owing to the concentration effect at $0.9 \mathrm{~nm}^{-1}<q<5 \mathrm{~nm}^{-1}$ and deviated upward for $q<0.8 \mathrm{~nm}^{-1}$ owing to the formation of branching. The branching was closely related to the sol-gel transition. These findings lead to the conclusion that the aggregated TRI functions in the manner of a semiflexible polymer chain in octanol and that the polymer-chain-like behavior of TRI cylinders, as well as the presence of branching, is the origin of the gel nature of this system. As far as we know, there has been no study to measure the scattering from cylindrically aggregated molecules over such a wide range of scattering vectors.

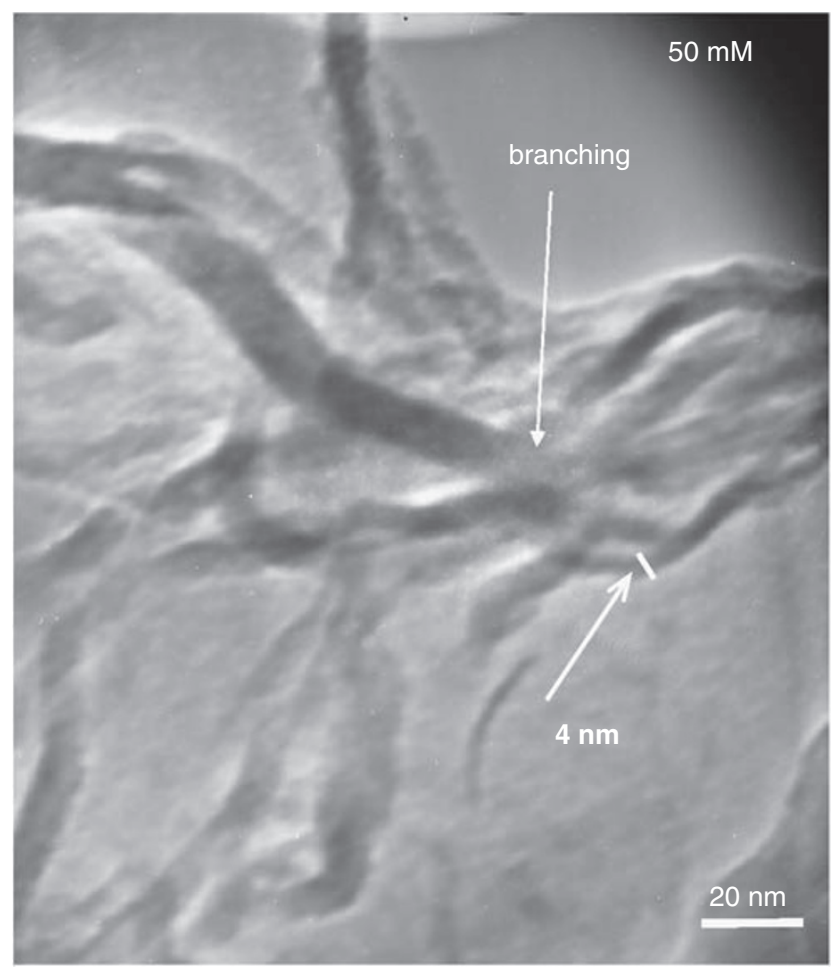

Figure 7 A typical TEM image of the TRI-C8/octanol gel.

\section{ACKNOWLEDGEMENTS}

We thank Dr S Sasaki for experimental help at the SPring-8 facility and Dr K Terao for discussions to determine the worm-like cylinder parameters from the Holzer plot. This work is financially supported by a Grant-in-Aid for Scientific Research (16350068 and 16655048) and by JST CREST. The synchrotron radiation experiments were conducted at SPring-8 BL40B2.

1 Weiss, R. G. \& Terech, P. Molecular Gels (Springer, Tokyo, 2006).

2 Terech, P \& Weiss, R. G. Low-molecular mass gelators of organic liquids and the properties of their gels. Chem. Rev. 97, 3133-3160 (1997).

3 Mukkamala, R. \& Weiss, R. Physical gelation of organic fluids by anthraquinone-steroidbased molecules. Structural features influencing the properties of gels. Langmuir 12, 1474 (1996). 
4 Terech, P. in Molecular Gels: Materials with Self-Assembled Fibrillar Networks, (eds Weiss R. G. \& Terech P.) (Springer, US, 2005), pp. 275.

5 Terech, P., Ostuni, E. \& Weiss, R. G. Structural study of cholesteryl anthraquinone-2 carboxylate (CAQ) physical organogels by neutron and $\mathrm{X}$-ray small angle scattering. J. Phys. Chem 100, 3759 (1996).

6 Jeong, Y., Friggeri, A., Akiba, I., Masunaga, H., Sakurai, K., Sakurai, S., Okamoto, S., Inoue, K. \& Shinkai, S. Small-angle X-ray scattering from a dual-component organogel to exhibit a charge transfer interaction. J. Colloid Interface Sci. 283, 113 (2005).

7 Jeong, Y., Hanabusa, K., Masunaga, H., Akiba, I., Miyoshi, K., Sakurai, S. \& Sakurai, K. Solvent/gelator interactions and supramolecular structure of gel fibers in cyclic bisurea/primary alcohol organogels. Langmuir 21, 586 (2005).

8 Sakurai, K., Jeong, Y, Koumoto, K., Friggeri, A., Gronwald, O., Sakurai, S., Okamoto, S., Inoue, K. \& Shinkai, S. Supramolecular structure of a sugar-appended organogelator explored with synchrotron X-ray small-angle scattering. Langmuir 19, 8211 (2003).
9 Terech, P., Furman, I. \& Weiss, R. G. Structures of organogels based upon cholesteryl 4(2-anthryloxy)butanoate, a highly efficient luminescing gelator: neutron and X-ray small-angle scattering investigations. J. Phys. Chem 99, 9558 (1995)

10 Ishi-i, T., Kuwahara, R, Takata, A, Jeong, Y, Sakurai, K \& Mataka, S An enantiomeric nanoscale architecture obtained from a pseudoenantiomeric aggregate: covalent fixation of helical chirality formed in self-assembled discotic triazine triamides by chiral amplification. Chemistry 12, 763 (2006).

11 Masunaga, H., Sasaki, S, Tashiro, K, Hanesaka, M, Takata, M, Inoue, K, Ohta, N \& Yagi, $\mathrm{N}$ Development of synchrotron DSC/WAXD/SAXS simultaneous measurement system for polymeric materials at the BL4OB2 in SPring-8 and its application to the study of crystal phase transitions of fluorine polymers. Polym. J. 39, 1281 (2007).

12 Roe, R.- J. Method of X-ray and Nutron Scattering in Polymer Science (Oxford University Press, Oxford, New York, 2000).

13 Nakamura, Y. \& Norisuye, T. Scattering function for wormlike chains with finite thickness. J Polym. Sci. Polym. Phys. Ed. 42, 1398 (2004).

Supplementary Information accompanies the paper on Polymer Journal website (http://www.nature.com/pj) 